\title{
El estatus sintáctico-semántico del caso dativo con verbos estativos latinos*
}

\author{
Concepción Cabrillana
}

\author{
Universidad de Santiago de Compostela \\ concepcion.cabrillana@usc.es
}

\section{The syntactic-semantic status of the dative case with Latin stative verbs}

El propósito de esta investigación es determinar la naturaleza sintáctico-semántica del dativo que aparece en algunas construcciones de tres verbos estativos latinos (maneo, permaneo y remaneo), los cuales comparten — entre otras — la noción semántica de «permanencia».

Tras ofrecer los datos proporcionados por los léxicos y por un estudio de corpus, se propone una diferenciación de contenidos semánticos y de esquemas de complementación de los verbos analizados. El examen más pormenorizado de las estructuras en que aparece un constituyente en dativo permite entender que la noción verbal presente en estas construcciones es de naturaleza existencial y que el dativo puede tener un papel argumental. De acuerdo con ello, se propone un marco predicativo de la estructura, así como una justificación del proceso de constitución de estas construcciones.

Palabras clave: dativo latino; construcciones existenciales; marco predicativo; preverbios; verbos estativos.
The aim of this article is to determine the syntactic-semantic nature of the dative appearing in some constructions of three Latin verbs (maneo, permaneo and remaneo) which share - among othersthe semantic notion of «permanence».

After presenting the data provided by the dictionaries and a corpus study, I propose different semantic and complementation structures for the verbs analysed. A more detailed examination of the structures in which a dative constituent appears, allows us to understand that the verbal notion of these constructions is existential in nature and that the dative can be considered an argument. Accordingly, I propose a predicate frame of the construction as well as a possible process for the setting of this construction.

Key words: Latin dative; existential syntactic structures; predicate frame; preverbs; stative verbs.

* Este estudio ha sido realizado en el marco del Proyecto de Investigación FFI201347357-C4-4-P («Problemas de rección en griego y en latín: verbos de estado y existencia») financiado por el Ministerio de Economía y Competitividad. Agradezco a los miembros de este Grupo de investigación las sugerencias que han realizado sobre una versión previa de este trabajo. Asimismo, doy las gracias a Ch. Lehmann y a uno de los revisores anónimos por las indicaciones que han aportado a este artículo. 
I. INTRODUCCIÓN: OBJETIVO, ESTRUCTURA Y CORPUS DEL TRABAJO

Este trabajo se enmarca dentro del que está llevando a cabo el Grupo de Investigación que elabora y estudia una Base de Datos sobre Rección y Complementación de verbos griegos y latinos (REGLA) ${ }^{1}$.

Desde el punto de vista metodológico el estudio se inscribe en el marco teórico funcionalista que ha adoptado este Grupo de investigación, y que considera el léxico del predicado como el factor predeterminante de sus condiciones sintácticas de uso: el número de los elementos obligatorios que requiere $^{2}$ y sus características léxicas y funcionales ${ }^{3}$.

El objeto concreto de esta investigación lo constituye el estudio de ciertas estructuras cuantitativamente marginales pero claramente presentes en construcciones de tres verbos estativos (maneo, permaneo y remaneo) que tienen en común la expresión de nociones semánticas similares, siendo la de «permanencia» la preponderante de manera global. Las construcciones que se tratarán son aquellas que presentan un constituyente en dativo, con el propósito de determinar el estatus sintáctico y el valor semántico de tal dativo, algo sobre lo que no existen conclusiones claras hasta el momento. Aunque no se trate de una estructura frecuente ${ }^{4}$, en el análisis lingüístico es importante describir y explicar no sólo lo que sucede de manera predominante sino que también hay que dar cuenta de la relevancia de que un hecho particular tenga lugar, aunque no sea de una forma mayoritaria.

El trabajo se estructura como sigue: se partirá de los datos proporcionados por los léxicos y por un estudio de corpus para proponer una diferenciación de contenidos semánticos y de esquemas de complementación de los verbos objeto de análisis que sirva de presentación panorámica de la realidad sintáctico-semántica de los mismos y de marco de referencia para el estudio que

${ }^{1}$ Cf. $<$ http://www.uam.es/proyectosinv/regula/index.html>.

${ }^{2}$ Lehmann (2002) matiza que la obligatoriedad de un argumento es una de las manifestaciones de la valencia del predicado, pero no la más importante.

${ }^{3}$ Para el modelo teórico que defiende la relación entre el significado del verbo y las propiedades de los constituyentes considerados obligatorios que acompañan al predicado, cf. Dik 1989, Van Valin y LaPolla 1997, Pinkster 2015. Algunas aplicaciones de este modelo pueden verse en Baños et al. 2003, Torrego et al. 2007. Por otro lado, se tendrán en cuenta aportaciones más concretas y pertinentes, realizadas por los trabajos de Lehmann 1983, Pinkster 1988 o García-Hernández 1996.

${ }^{4} \mathrm{Y}$ de ahí que las cantidades finales con las que se trabaja sean escasas, a pesar de que se ha expurgado un corpus amplio (cf. Tablas 1 y 5 y nota 52 ). 
aquí se pretende $(\S 2)$. A continuación se abordará el examen más pormenorizado de las estructuras en que aparece un constituyente en dativo, tanto en las formas preverbadas $(\S 3.1)$ como en el verbo simple correspondiente (§3.2). Se propondrá (i) un mecanismo de verificación de las propuestas que se realizan para las construcciones analizadas (§ 4) y (ii) un esquema de complementación para las mismas, así como (iii) una probable justificación del proceso de constitución de estas estructuras (§5).

El corpus fundamental utilizado en este trabajo es el que se encuentra previamente analizado en la Base de Datos de REGLA, y que resulta suficientemente representativo de géneros y épocas literarias: Cato (Agr.), Plaut. (Amph., Asin., Aul., Bacch., Capt., Cas., Curc., Epid.), Caes. (Gall.), Sall. (Cat., Iug.), Cic. (Verr., Catil., S. Rosc., Mil., Mur., Sest., Dom., Off.), Ou. (Met.), Liu. 1-10, Colum., Plin. (Paneg.), Plin. (Nat.), Tac. (Ann.), Sen. (Dial. 6, 12; Epist.), Petron. No obstante, y para el estudio de puntos determinados a lo largo del análisis, los datos que proporciona este corpus se complementarán, en un primer momento, con los correspondientes a Lucrecio, Virgilio (Aen.), Lucano y Estacio (Theb.) por las razones que se indicarán en su momento. Para el tratamiento de algunos puntos concretos, el corpus se extenderá al resto de la producción tacitea (Hist., Agr., Dial., Germ.) y virgiliana (Eclg., Georg.), y a todas las demás obras poéticas recogidas en la base de datos de LASLA ${ }^{5}$.

\section{DifERENCIACIÓN GENERAL DE CONTENIDOS SEMÁNTICOS Y VALENCIAS CUANTITATIVAS}

Como cuestión previa, conviene aclarar que la expresión del contenido semántico compartido por maneo, permaneo y remaneo no está exenta de problemas, porque bajo esa noción genérica y común de «permanencia» existen matices de significado que conllevan el establecimiento de estructuras de complementación diferentes. Y precisamente uno de los problemas lo constituye la propia diferenciación, en ocasiones, del contenido semántico concreto que el verbo expresa - permanencia en un lugar o en un estado, permanencia de una cualidad, de la existencia, etc.-, lo cual, como se ve con detalle en

${ }^{5}$ Cf. $\S 3.2$ y Tablas 4 y 5. Las obras incluidas en ese corpus del Laboratoire d'Analyse Statistique des Langues Anciennes son: Catull.; Hor., Ars, Carm., Epist., Epod., Sat., Carm. saec.; Iuu.; Ou., Am., Ars, Epist., Fast., Hal., Ib., Medic., Pont., Rem., Trist.; Pers.; Prop.; Sen., Ag., Herc., Herc. O., Med., Oed., Paedr., Phoen., Thy., Tro. y Tib. 
otro trabajo ${ }^{6}$, da lugar a esquemas sintácticos distintos. Baste aquí sólo proponer una presentación de esos contenidos semánticos, de la asignación de su valencia cuantitativa y mostrar su frecuencia de empleo.

Así, si se parte de los datos proporcionados por los léxicos al uso ${ }^{7}$ y se combinan estos con los que arroja el análisis del primer corpus mencionado en $\S 1$, podrían distinguirse en principio tres contenidos semánticos de estructura bivalente y uno de esquema monovalente:

- Bivalente:

- «permanecer en $\mathrm{x}^{8}$ »

- «continuar siendo $\mathrm{x} »$

- «esperar x» (sólo presente en maneo)

- Monovalente:

- «continuar existiendo/subsistir»

Aunque el número de apariciones no es muy alto, especialmente en los preverbados, se ofrecen también a continuación las cantidades relativas de los tipos distinguidos para poder contar con datos que permitan ofrecer una panorámica de la frecuencia de uso de los verbos y de los contenidos semánticos presentes en ellos:

Tabla 1. Verbos y número de ocurrencias según acepciones y estructuras sintácticas en el corpus de REGLA

\begin{tabular}{|l|r|r|r|c|}
\hline \multicolumn{1}{|c|}{ Verbo } & $\begin{array}{c}\text { «permanecer } \\
\text { en } \mathbf{x}\end{array}$ & \multicolumn{1}{|c|}{ «subsistir» } & $\begin{array}{c}\text { «continuar } \\
\text { siendo x» }\end{array}$ & $\begin{array}{c}\text { «esperar } \mathbf{x » ~} \\
\text { (tr.) }\end{array}$ \\
\hline maneo $(349 \mathrm{x})$ & $192(55,01 \%)$ & $88(25,21 \%)$ & $52(14,89 \%)$ & $17(4,89 \%)$ \\
\hline permaneo $(68 \mathrm{x})$ & $32(47,05 \%)$ & $20(29,41 \%)$ & $16(23,54 \%)$ & - \\
\hline remaneo $(81 \mathrm{x})$ & $74(91,35 \%)$ & $5(6,17 \%)$ & $2(2,48 \%)$ & - \\
\hline TOTAL $(498 \mathrm{x})$ & $298(59,83 \%)$ & $113(22,69 \%)$ & $70(14,05 \%)$ & $17(3,43 \%)$ \\
\hline
\end{tabular}

Como se ve, la acepción mayoritaria en los tres verbos es la de naturaleza en principio locativa («permanecer en algún lugar») — de estructura bivalente- - seguida a bastante distancia por la existencial monovalente («subsistir, continuar existiendo») y por la copulativa («continuar siendo de alguna ma-

${ }^{6}$ Cabrillana 2015.

7 Thesaurus Linguae Latinae (ThLL), Oxford Latin Dictionary (OLD), Gaffiot, Forcellini.

${ }^{8}$ Representa aquí el segundo argumento (A2) de la construcción. 
nera»), de nuevo bivalente. Sólo en el caso de maneo existe una construcción transitiva («esperar algo [a alguien]»); curiosamente, y frente a la tendencia observada ${ }^{9}$ de que la preverbación cataliza el proceso de transitivación en la lengua latina, ninguno de los preverbados muestra ese tipo de construcción ${ }^{10}$.

Ejemplos de las cuatro construcciones reflejadas en la Tabla 1 se presen$\tan$ respectivamente en (1)-(4):

- «permanecer en $\mathrm{x} »$ :

(1) dixit C. Causinius Schola, ..., P. Clodiumillo die in Albanomansurum fuisse (Cic., Mil. 46)

Gayo Causinio Escola, ..., manifestó que Publio Clodio tenía la intención de quedarse aquel día en Alba ${ }^{11}$

- «continuar existiendo/subsistir»:

(2) Tiberius enim Gracchus laudabitur, dum memoria rerum Romanarum manebit (Cic., Off. II 43)

la gloria de Tiberio Graco, hijo de Publio, durará mientras se conserve la memoria del Imperio Romano

- «continuar siendo x»:

(3) reliquum anni quietum ab urbanis motibus et ab externis mansit (Liu. III 72.7) durante el resto del año reinó la calma, sin perturbaciones interiores ni exteriores

- «esperar $\mathrm{x} »$ :

(4) intus illa te, si se arcessas, manet (Plaut., Cas. 542)

está dentro, esperando a que vengas a buscarla ${ }^{12}$

${ }^{9}$ Cf. Ernout y Thomas 1953, p. 20-21; Álvarez H. 2009, p. 133 ss.

${ }_{10}$ Sobre la rareza de la transitivación en verbos de estado inacusativos, cf. Cabrillana 2011. Lehmann 1983 señala que esa tendencia a la transitivación está ligada a determinadas condiciones.

${ }^{11}$ Las traducciones, excepto cuando se señale lo contrario y aún a riesgo de que no siempre reflejen adecuadamente desde un punto de vista sintáctico-semántico las construcciones que en cada caso interpreten, están tomadas de las correspondientes a la serie de Gredos. En el caso de Stat. Theb. - y a falta de una traducción castellana actualizada y fiable-, se proporcionan las traducciones inglesas de la colección de Loeb Classical Library (D. R. Shackleton Bailey 2003: Statius. Thebaid, 1-7, Cambridge (Mass.) - Londres: Harvard University Press). No se ofrecen traducciones personales con el fin de no condicionar la interpetación del texto latino.

${ }^{12}$ Las traducciones de Plauto están tomadas de J. R. Bravo 1993-2005: Plauto. Comedias, I-II. Madrid, Cátedra. 


\section{ANÁlisis DE LAS ESTRUCTURAS CON DATIVO}

\subsection{Dativo con preverbados}

En el trabajo más amplio ya aludido ${ }^{13}$ sobre los verbos estativos que albergan entre sus contenidos semánticos el de «permanencia» se hace referencia detalladamente a dos estructuras que muestran en ocasiones una difícil distinción: las existenciales y las locativas cuyo relatum puede tener distintas codificaciones, mayoritariamente con constituyentes que tienen una connotación local; estos son - por orden de frecuencia - sintagmas preposicionales (Sprep), ablativos o adverbios con valor claramente locativo, locativos propiamente dichos, así como oraciones introducidas por un elemento con valor de ubicación ${ }^{14}$.

Adicionalmente, como se ha anunciado, entre los elementos que aparecen con los verbos estudiados se cuentan también casos en los que aparece un dativo. A este respecto, Lehmann 1983, p. 156 señala que algunos preverbios nunca o casi nunca tienen su relatum en dativo; más concretamente, el autor (Lehmann 1983, p. 156) no incluye re- entre los preverbios que admiten un relatum en dativo ${ }^{15}$. Por su parte, Pinkster 2015, pp. 1192-1193. ${ }^{16}$ tampoco incluye este tipo de verbos entre aquellos con nociones semánticas que utilizan el dativo como A2, excepción hecha del caso que se comentará más adelante ${ }^{17}$ para el verbo maneo. Así pues, ¿cómo entender, clasificar y caracterizar estas estructuras?

Comencemos por analizar los casos que muestran dativo con los preverbados; se trata ciertamente de escasos ejemplos con permaneo y remaneo:

(5) nam nisi hoc fecerimus, ne uirtus quidem nobis permanebit, quae exercendo sensu ualet (Sen., Epist. 109.15)

porque, si tal no hiciéramos, tampoco permanecería firme en nosotros la virtud que mantiene el vigor ejercitándose

${ }^{13}$ Cabrillana 2015.

${ }^{14}$ En relación con ello, y en una afirmación que aproxima su apreciación al caso concreto de los verbos que aquí se analizan, Lehmann 1983, p. 147 afirma que «almost all of the Latin preverbs have a basic local meaning». Además, el autor da cuenta de que expresiones locales o temporales pueden estar implícitas o no expresas, también con verbos preverbados (Lehmann 1983, p. 150), algo que se hace más frecuente en el caso de que el preverbio no tenga un significado concreto.

${ }^{15}$ Ahora bien, de lo que expresa este autor se desprende que si el verbo simple rige un dativo, la preverbación no propicia cambios en ese aspecto.

${ }^{16}$ Agradezco a H. Pinkster haberme dado la oportunidad de leer este material, no publicado en el momento de escribir este artículo.

${ }^{17}$ Cf. $\S 3.2$. 
(6) inde mihi quaedam permansere, Lucili (Sen., Epist. 108.15) de aquella época he conservado ciertos propósitos, Lucilio

(7) foliorum unitas in suo cuique genere permanet, praeterquam populo, hederae, crotoni, quam et cici diximus uocari (Plin., Nat. XVI 47) 18 $^{18}$ cada árbol, dentro de su especie, tiene hojas idénticas, excepto el álamo, la hiedra y el ricino, que hemos dicho que también se llama 'cici'

(8) arbor ipsa in Europae citeriore caelo, ..., primum cerceis in Elpenoris tumulo uisa traditur graecumque ei nomen remanet (Plin., Nat. XV 39) ${ }^{19}$ el propio árbol, en el horizonte de la Europa citerior..., cuenta la tradición que fue visto por primera vez en la tumba de Élpenor en el Circeo, y le queda su nombre griego

(9) et uobis aeterna sollicitudo remanebit (Sall., Iug. 31) y a vosotros os quedará para siempre la inquietud

Ahora bien, no en todos estos ejemplos está del todo claro, al menos a primera vista, si los constituyentes en dativo son realmente un tipo de relatum argumental o se encuentran a nivel de satélite en la periferia ${ }^{20}$; ni siquiera es meridiano si se trata de casos en los que debamos inclinarnos por una acepción plenamente locativa («permanecer en $\mathrm{x} »)$ o si reflejaría mejor la idea del autor una noción existencial («subsistir [para $\mathrm{x}$ ]»): la preferencia por la opción local — si bien en un sentido no físico- podría parecer más clara en (5) a partir de la interpretación que el traductor hace

18 Sobre la presencia de dativos afectados por el proceso verbal de naturaleza no humana y no animada en Plinio, cf. Pinkster 2005, pp. 242-243; el autor lo ve como un rasgo congruente con la naturaleza no antropocéntrica de la obra pliniana. Pinkster 2005, pp. 243-244 reconoce también el frecuente uso de la omisión de elementos obligatorios en Plinio ('zero-anaphora') y que pueden ser sobreentendidos a partir del contexto (precedente).

${ }^{19}$ Una de las conclusiones de Lehmann 1983, p. 160 cuando alude a los preverbados es que «preverbs do not affect the valency of the verb in a regular way». En efecto, así parece de manera general en el caso de los verbos analizados; de hecho, existen contextos paralelos a este ejemplo en maneo: cf. ej (14b).

${ }^{20}$ Pinkster 2015, p. 893 reconoce la dificultad de decidir en ocasiones sobre la cualidad argumental o no (i.e., considerándolos como un adjunto beneficiario) de algunos dativos. 
del texto; sin embargo, si se considera esa opción como la más probable, habría que admitir la posibilidad de que en el marco predicativo de la noción locativa aparezcan dativos personales en alternancia con complementos de Ubicación que suelen expresarse mediante un sintagma preposicional, un adverbio o un constituyente en ablativo o locativo, además de que muy probablemente, la Función Semántica sería distinta. Esa posibilidad no queda claramente recogida en los léxicos; la constatación más cercana se encuentra en ThLL, permaneo, 1528,1, pero los escasísimos ejemplos son sólo tardíos (ss. III-V d.C.) y no del todo seguros ${ }^{21}$. La otra posibilidad - esto es, interpretar la noción del verbo como existencialaparece algo más explícitamente recogida en el ThLL, permaneo, 1526,2, con algún ejemplo de épocas clásica e imperial ${ }^{22}$.

$\mathrm{Y}$ es que, en efecto, hay que hacer notar que en verbos con posible acepción existencial, la construcción con dativo resulta normal y es equivalente desde el punto de vista estructural y semántico al empleo existencial-posesi$\mathrm{vo}^{23}$. Esta estructura parece especialmente clara en (6), y podría postularse también para (7): aunque curiosamente aparece en ese ejemplo un sintagma preposicional (in suo genere), éste no parece tener función argumental de Ubicación del S(ujeto) (foliorum unitas), mientras que sí podría postularse dicha función argumental para el dativo cuique, de nuevo candidato - por tanto- a ser el A2 de una estructura existencial-posesiva. Lo mismo podría decirse de (8)-(9); ciertamente, en este último ejemplo, cabría la duda de que el pronombre uobis fuera aquí un ablativo sin preposición, pero los dos únicos casos del corpus analizado en los que remaneo aparece con un pronombre

${ }^{21}$ Los ejemplos son los siguientes: Acta christiana, Petr. 1: quartus permansit Paulo, ut ubi uellet iret ab urbe [permisit Turner]; Acta conciliorum oecumenicorum (sc. Epheseni a. 431 [tom. I]: Hier., Tract. p. 557 I,45: confessa es, quod mihi permaneres; Acta conciliorum oecumenicorum (sc. Epheseni a. 431 [tom. I]: I I, 3 p. 29,4: ei Nestorio permanerent (sc. consentientes).

${ }^{22}$ Cf., vg., Cic., Parad. 17 (huic nihil potest esse certi, nihil, quod exploratum habeat permansurum sibi unum diem); Cic., Fin. II 106 (quo modo id potuit mortuo permanere?); Lucan. VIII 664 (permansisse decus sacrae venerabile formae).

${ }^{23}$ Cf. Rosén 1998, p. 735; Cabrillana 2003, pp. 83-84; 2010, p. 94, y la bibliografía ahí señalada. La utilización de la etiqueta «posesivo/a» viene justificada más por razones de claridad expositiva que por motivos teóricos. Un ejemplo que ilustra este tipo de construcciones con el verbo sum sería el siguiente: sex filii nobis, duae filiae sunt, utraeque iam nuptae («tenemos seis hijos y dos hijas, ambas ya casadas», Liu. XLII 34.4). 
como relatum lo hace acompañado de preposición; se trata de los ejemplos siguientes:

(10a) remanet nitor unus in illa (Ou., Met. I 552)

en ella permanece sólo su belleza ${ }^{24}$

(10b) de quo si uos seuere ac religiose iudicaueritis, auctoritas ea, quae in uobis remanere debet, haerebit (Cic., Verr. I 3)

si vosotros lo juzgáis con severidad y escrúpulo, quedará bien asegurada la autoridad que en vosotros debe permanecer

Aunque sólo se utilizara aquí un criterio de paralelismo en el uso, al menos en el corpus estudiado, es más verosímil que uobis sea, en efecto, un dativo.

De este modo y aunque, como se ha dicho, en la obra más reciente de Pinkster 2015, pp. 1192-1193 no se considera que el dativo de estos casos pueda ser A2, creo que, en cierto modo, este dativo resulta paralelo al que aparece eventualmente con verbos como sum cuando se utiliza con su noción existencial $^{25}$; en ese sentido - y esto es lo que aquí se propone- sí tendría naturaleza argumental, porque, de hecho, la noción que parece subyacer en estos usos de permaneo y remaneo es en muchos casos la de una posesión, más y menos prototípica ${ }^{26}$, continuada en el tiempo. Por consiguiente, en estos ejemplos podríamos estar no tanto ante casos de relatum en dativo personal $^{27}$, sino más bien ante el A2 eventualmente actualizado de estructuras existenciales-posesivas ${ }^{28}$, — eso sí, de manera poco frecuente y siempre en el caso de los preverbados, con el primer argumento (A1) [-concreto].

${ }^{24}$ Las traducciones de esta obra corresponden a C. Álvarez y R. M. Iglesias 1995 (2005): Ovidio. Metamorfosis, Madrid, Cátedra.

${ }^{25}$ Cf. Pinkster 1995, pp. 27-28; cf. también http://www.uam.es/proyectosinv/regula/mpla/ sum.pdf.

${ }^{26}$ Cf. Seiler 1983, Cabrillana 2003, Baldi y Nuti 2010.

${ }^{27}$ La tendencia a que el dativo exprese un complemento personal parece clara, también con preverbados; cf., p.ej., Longrée 2005, pp. 297, 299, quien lo ve como una muestra de búsqueda de variatio en la expresión de la complementación. El estudio de Longrée se realiza, entre otros verbos, sobre algunos compuestos de cedere, verbos para los que se reconocen también usos absolutos (v. p. 298).

${ }^{28}$ Esta interpretación cuenta, además, con apoyos tipológicos: cf., entre otros, Blake 2001, pp. 145, 150-151; Næss 2009, pp. 572-575. 
Un argumento más a favor de la consideración propuesta para estas estructuras es la dificultad para entender estos dativos como adnominales. En efecto, ese tipo de dativos es propio de construcciones en las que un dativo determina a un adjetivo o a un sustantivo que generalmente se encuentra en relación (semántica) más o menos directa con verbos que poseen un segundo argumento en dativo ${ }^{29}$; no es éste el contexto de las estructuras objeto de estudio ${ }^{30}$.

\subsection{Dativo con verbo simple}

En relación a la postura de Lehmann a la que se aludía más arriba —que el dativo nunca o casi nunca aparece como relatum de preverbados-, hay que matizar que el lingüista alemán 1983, p. 158 entiende el dativo en esos usos (i.e., como caso que expresa el presunto relatum) como un empleo que constituye una extensión de su función propia, «which is to express an object indirectly participating in an action or process». En una línea parcialmente similar, García-Hernández 1996, p. 34, refiriéndose a la alternancia acusativo/ dativo $^{31}$ como régimen argumental en simples y preverbados, señala que «la diferencia entre la construcción con acusativo y con dativo depende ante todo de si el término de la acción se concibe como objeto o como destinatario, lo que normalmente confiere un carácter menos directo a la construcción con dativo». Además, el autor 1996, p. 36 advierte de que «los poetas incluso

${ }^{29}$ Cf. Baños 2009, pp. 196-197, Pinkster 2015, pp. 217-219, 1074-1075, 1217-1218. Por otro lado, es presumible que la presunta adnominalidad de estos dativos hiciera de ellos elementos más prescindibles que en el caso de los argumentales.

${ }^{30}$ La estructura estaría presente en casos como el de Ou., Am. I 10.46: omnia conductor soluit; mercede soluta / non manet officio debitor ille tuo («el comprador lo ha pagado todo cuando ha pagado el precio: aquél ya no queda como un deudor a tu servicio»). En efecto, el verbo debeo, del que procede debitor (cf. Ernout y Meillet 1932, s. u. debeo), se puede construir con dativo; cf. también OLD, debitor, 1a. Otro ejemplo claro lo constituye Ou., Ars I 134, en este caso en una construcción copulativa con el adjetivo correspondiente como Complemento del Sujeto: scilicet ex illo sollemnia more theatra / nunc quoque formosis insidiosa manent («lo cierto es que desde entonces, por costumbre inveterada, los teatros siguen siendo también ahora lugares de asechanza para mujeres hermosas»).

${ }^{31}$ Una cuestión interesante la constituye el estudio de contextos de maneo en los que pudiera rastrearse una cierta relación alternante entre la construcción con dativo y la que se hace con acusativo: cf. p.ej., Ou., Met. XI 540: non tenet hic lacrimas, stupet hic, uocat ille beatos, / funera quos maneant («éste no retiene lágrimas, éste está atónito; aquél llama felices a los que les está reservado un entierro»). Esta cuestión se abordará en otro lugar. 
confirman su preferencia por la expresión directa empleando el dativo allí donde se esperaría un caso preposicional». A este respecto, hay que señalar que en el caso de permaneo y remaneo, todos los ejemplos detectados en el corpus corresponden a textos en prosa $^{32}$. Cabe la posibilidad de que sea la mayor plenitud semántica atribuible a los preverbados ${ }^{33}$ la que precisamente motive la menor presencia del constituyente en dativo.

El investigador español (García-Hernández 1996, p. 37) continúa apuntando que «este dativo que supone cierta animación de conceptos inanimados (tectis, stabulis), no deja de darse también con los verbos simples». En efecto, la presencia de dativos con maneo se constata en el corpus en un total de 21 ocasiones $(=6,01 \%$ de sus apariciones totales). A este respecto, Pinkster 2015 , p. 129 - ahora sí- deja constancia de que maneo puede tener como A2 un dativo con el sentido de «to be in store»" («lo que le espera/se le reserva [a alguien]») y ese podría ser el sentido de ejemplos como $(11)^{35}$ :

(11) malis una uoluptas est et haec breuis, dum accipiunt beneficia, ex quibus sapienti longum gaudium manet ac perenne (Sen., Epist. 81.24)

a los malos les alcanza un solo placer, y por cierto efímero, mientras reciben los beneficios; de éstos aguarda al sabio un gozo prolongado y perenne

Ahora bien, en la gran mayoría de los casos, la noción semántica que se observa como subyacente a la construcción con dativo es más bien la de «conservar alguien algo $»^{36}$, y en ese sentido, es de nuevo la acepción existencial-posesiva, y no la locativa, la que parece la más próxima. Esto es lo que

${ }^{32}$ Una investigación adicional en Lucrecio tampoco confirma la existencia de relatum en dativo con los dos preverbados; con respecto al caso excepcional de Lucr. III 402 (at manet in uita cui mens animusque remansit («en cambio se mantiene en la vida aquel a quien mente y espíritu se le retienen»), cf. comentario en Cabrillana 2015, § 2.2.3). Por su parte, otro texto poético de época augústea, la Eneida virgiliana, no registra ocurrencias de permaneo ni de remaneo.

${ }^{33}$ Cf. Cabrillana 2015.

${ }^{34}$ Cf. también OLD, s. u., 4, aunque los ejemplos de esa acepción que ofrece el léxico aparecen o bien construidos con acusativo o bien en sentido absoluto; sólo en uno de ellos el verbo aparece con dativo: cuius ... tibi fatum ... manet («cuyo ... destino te ... es reservado», Cic., Phil. II 11).

${ }^{35}$ Pese a la interpretación realizada por el traductor correspondiente en este ejemplo (cf. lo señalado en nota 11), la consideración del ejemplo en su contexto permite pensar que la permanencia a la que aquí se apela es, en efecto, una permanencia real y no virtual.

${ }^{36}$ Nótese, por otro lado, que en el ejemplo proporcionado se da una suerte de paralelismo entre la construcción que aparece en la primera oración —sum + dativo - y la que se da en 
se desprende de los datos del ThLL cuando hace referencia a la aparición de estructuras con dativo:

(12a) indicatur, cui (rei) aliquid esse pergat: animantibus (ThLL, maneo, 286,16 ss., 44 ss.)

Esta acepción la incluye a su vez este diccionario en la idea existencial de la construcción intransitiva, que el Léxico expresa del siguiente modo:

(12b) de diuturnitate status i. q. durare, in condicione, statu per aliquod tempus perseverare; non raro diuturnitas magis ita respicitur, ut significetur aliquid (aliquem) post longum tempus adhuc exstare, superesse (ThLL, maneo, 284,37 ss.)

Así, especialmente en los historiadores, es frecuente este tipo de construcción cuando se quiere significar que los miembros de alguna magistratura siguen poseyendo determinada autoridad o poder:

(13a) nondum maturus imperio Ascanius Aeneae filius erat; tamen id imperium ei ad puberem aetatem incolume mansit (Liu. I 3.1)

Ascanio, el hijo de Eneas, no estaba aún maduro para el poder, pero este poder se le conservó sin merma hasta la pubertad

(13b) mansitque consulibus potestas deligendi, donec eum quoque honorem populus mandaret (Tac., Ann. XI 22.4)

además, conservaron los cónsules el poder de escogerlos hasta que también tal magistratura pasó a conferirla el pueblo

Por otro lado, lo que se conserva puede ser también otra realidad prácticamente siempre abstracta ${ }^{37}$ : un recuerdo (14a), un nombre (14b), un sentimiento (14c), una forma (14d), etc. Algunos ejemplos pueden hacer dudar de nuevo si se está ante una estructura existencial-posesiva o ante una construcción locativa, donde el relatum personal ${ }^{38}$ se codificaría en dativo ( ${ }^{2}$ ?), pero ello se debe más bien a una cuestión de interpretación. Así, y sin que sea del

la subordinada de relativo - maneo + dativo-; en efecto, a través de ambas construcciones se proporciona un contraste en una misma línea semántica.

37 Concretamente, 19x con A1 abstracto y $2 \mathrm{x}$ donde el A1 es un evento.

3820 casos contienen claramente como rasgo [+humano] y el restante (Tac., Ann. XIV 51.2) es asimilable a ellos, ya que se trata de una realidad personificada, ciuitati, que designa a los habitantes de la ciudad. 
todo posible tener una certeza absoluta, lo más probable es que se trate de estructuras existencial-posesivas:

(14a) memoriam eius irae Tusculanis in poenae tam atrocis auctores mansisse ad patrum aetatem constat (Liu. VIII 37.12)

es un hecho comprobado que permaneció en la memoria de los tusculanos el resentimiento contra quienes propusieron un castigo tan atroz hasta la época de nuestros padres

(14b) aliqua et mihi gratia ponto est, / .. / ... Graiumquemanet mihi nomen ab illa (Ou., Met. IV 481) $)^{39}$

también tengo yo [sc. Venus] alguna influencia sobre el mar, ... y permanece en mí el nombre griego de ella

(14c) manet quidem tibi, Marcia, etiamnunc ingens tristitia (Sen., Dial. VI 8.2) sin duda persiste aún en ti, Marcia, una inmensa tristeza

(14d) nulli sua forma manebat (Ou., Met. I 5) para nadie permanecía su propia figura

Nótese el paralelismo presente en las dos oraciones coordinadas de $(14 b)^{40}$, puesto que ambas responderían a la misma estructura existencial-posesiva.

En cuanto a la aludida preferencia de los poetas por la utilización del dativo - García-Hernández 1996, p. 36-, los datos podrían confirmarlo, aunque son escasos: en el corpus de REGLA, 15 casos $(=71,42 \%)$ corresponden a $\operatorname{prosa}^{41}$ y $6(=28,58 \%$ ) a poesía, todos ellos de Ovidio (43 a.C.-17 d.C.). Pero estos datos no son ilustrativos si no se ponen en relación con la totalidad de los ejemplos de esos autores recogidos en el corpus, ya que en éste, la prosa es más abundante que la poesía ${ }^{42}$. Así, la tabla siguiente mues-

${ }^{39}$ Cf. también Liu. I 3.7: ab eo coloniae aliquot deductae, Prisci Latini appellati. Mansit Siluiis postea omnibus cognomen, qui Albae regnarunt («puso éste [sc. Latino Silvio] en marcha algunas colonias, cuyos componentes se llamaron 'antiguos latinos'. Les quedó, en adelante, el apelativo de Silvio a todos los que reinaron en Alba»).

${ }^{40}$ Un paralelismo semejante se observaba también en (11).

${ }^{41}$ Livio, Plinio el Joven, Tácito y Séneca.

${ }^{42}$ Hay que tener en cuenta que también al género poético pertenecen los casos de Plauto en el corpus (45x), pero ninguno de ellos muestra dativo: 41 casos se adscriben a la acepción de «permanecer en $\mathrm{x}$ » $\mathrm{y}$ los otros 4 a la transitiva de «esperar $\mathrm{x}$ ». Por consiguiente, no pueden incluirse en el número de apariciones que aquí se computan. Con respecto a las peculiaridaders de estos casos, cf. Cabrillana 2015, § 2.2.2. 
tra la cantidad absoluta y relativa de las construcciones con dativo en los autores mencionados con respecto a la totalidad de ejemplos que de ellos recoge el corpus:

Tabla 2. Proporción de construcciones de maneo con dativo en el corpus de REGLA

\begin{tabular}{|l|c|c|}
\hline \multicolumn{1}{|c|}{ Autor-corpus } & N. $^{\mathbf{0}}$ total de ejemplos & N. $^{{ }^{0}}$ de construcciones con dativo \\
\hline Liu. I-X & 58 & $4(6,89 \%)$ \\
\hline Tac., Ann. & 56 & $7(12,50 \%)$ \\
\hline Sen., Epist.; Dial. VI; XII & 38 & $3(7,89 \%)$ \\
\hline Plin., Paneg. & 9 & $1(11,11 \%)$ \\
\hline TOTAL prosa & 153 & $15(9,80 \%)$ \\
\hline Ou., Met. & 54 & $6(11,11 \%)$ \\
\hline TOTAL GLOBAL & 207 & $21(10,14 \%)$ \\
\hline
\end{tabular}

En efecto, la proporción en el único poeta que presenta construcciones con dativo es algo mayor $(11,11 \%)$ que la que exhibe el conjunto de los textos en prosa $(9,80 \%)$ en esa misma situación, si bien la proporción es similar a la que aparece individualmente en Tácito ${ }^{43}$; en cualquier caso, la escasez de los datos aconseja tomar estas cantidades con cautela. Por ello, sería necesario comprobar si esta tendencia se confirma, examinando lo que ocurre al menos en poetas de épocas tanto clásica como imperial, de modo que sea posible realizar una comparación más proporcionada y homogénea con los datos ya $\operatorname{mostrados}^{44}$. Un criterio posible para seleccionar autores y obras comparables es el de la relativa afinidad en el género literario; de acuerdo con ello, las obras épicas de Virgilio (70-19 a.C.), Lucano (30-65 d.C.) y Estacio (45-96

${ }^{43}$ Este hecho puede sugerir que, en efecto, este historiador se acerca al uso que hacen los poetas de la estructura en cuestión, dada su particular forma de manejar y explotar las posibilidades de la sintaxis. De hecho, si se extiende la investigación al resto de su obra, la proporción de aparición de construcciones en dativo se mantiene muy similar: de un total de 51 apariciones de maneo en Germ. (6x), Agr. (5x), Dial. (2x) e Hist. (38x), se encuentran 6 estructuras con dativo, lo que constituye un $11,76 \%$; así, un cómputo global de toda la obra tacitea revela que de un total de 107 apariciones de maneo, en 13 ocasiones la construcción se realiza con dativo, lo que supone un $12,14 \%$.

${ }^{44}$ En el sentido, al menos, de lograr un mayor equilibrio entre el número total de apariciones en prosa y en verso. 
d.C.) parecen textos apropiados. Estos autores no se encuentran en el corpus inicial de la Base de datos de REGLA, por lo que se ha realizado una investigación adicional. Con ella, el número de casos de maneo analizados en total pasa de ser 349 (cf. Tabla 1) a alcanzar la cifra de $438^{45}$. Los datos se revelan en cierto sentido dispares: en primer lugar, el número de ocurrencias de maneo es casi el doble en Virgilio del que aparece en Lucano, hecho que puede condicionar la estadística final, tratándose además de una construcción de por sí poco frecuente. Por otro lado, la proporción de construcciones con dativo en Estacio - el poeta más adelantado en el tiempo- es hasta ahora la mayor, mientras que en Lucano es prácticamente inexistente. En todo caso, podría haber indicios para pensar que, en efecto, en términos globales, la construcción con dativo es algo más frecuente en poesía que en prosa.

Tabla 3. Proporción de construcciones de maneo con dativo en el corpus de REGLA, Virgilio (Aen.), Lucano y Estacio (Theb.).

\begin{tabular}{|l|c|c|}
\hline \multicolumn{1}{|c|}{ Autor-corpus } & N. $^{\mathbf{0}}$ total de ejemplos & N. $^{\mathbf{0}}$ de construcciones con dativo \\
\hline TOTAL corpus prosa & 153 & $15(9,80 \%)$ \\
\hline Ou., Met. & 54 & $7(12,96 \%)$ \\
\hline Verg., Aen. & 44 & $7(15,90 \%)$ \\
\hline Lucan. & 22 & $1(4,54 \%)$ \\
\hline Stat., Theb. & 23 & $5(21,73 \%)$ \\
\hline TOTAL poesía & 143 & $20(13,98 \%)$ \\
\hline TOTAL GLOBAL & 296 & $35(11,82 \%)$ \\
\hline
\end{tabular}

Con todo, y a pesar de haber ampliado el corpus de acuerdo con unas coordenadas genéricas y diacrónicas lógicas, las cantidades siguen siendo poco significativas, de forma que parece necesaria una investigación mayor. Por ello, voy a extender el análisis al resto de la obra tacitea (Hist., Agr., Dial., Germ.), ya que Tácito es uno de los prosistas que mayor densidad de construcciones con dativo utiliza; además, y por razones similares, voy a completar el análisis del resto de la producción virgiliana (Ecl., Georg.). Por último, examinaré todo el corpus poético que recoge la base de datos de

45 Este número de ejemplos se reparte del siguiente modo: corpus inicial analizado en la Base de datos de REGLA: 349x; Virgilio (Aen.): 44x; Lucano: 22x; Estacio (Theb.): 23x. 
LASLA; de esta manera, el número total de ejemplos analizados de maneo asciende a 481. En la Tabla 4 figuran las cantidades totales, subsumiendo las que aparecían en la Tabla 3:

Tabla 4. Proporción de construcciones de maneo con dativo en corpus ampliado ${ }^{46}$.

\begin{tabular}{|l|c|c|}
\hline \multicolumn{1}{|c|}{ Autor-corpus } & N. $^{\mathbf{0}}$ total de ejemplos & N. $^{\mathbf{0}}$ de construcciones con dativo \\
\hline \multicolumn{1}{|c|}{ TOTAL corpus prosa } & $204^{47}$ & $21(10,29 \%)$ \\
\hline Catull. & 11 & $2(18,18 \%)$ \\
\hline Verg. & 51 & $8(15,68 \%)$ \\
\hline Iuu. & 0 & 0 \\
\hline Pers. & 1 & $1(100 \%)$ \\
\hline Tib. & 8 & 0 \\
\hline Prop. & 13 & $1(7,69 \%)$ \\
\hline Hor. & 20 & $2(10,00 \%)$ \\
\hline Ou. & $103^{48}$ & $9(8,73 \%)$ \\
\hline Sen., Trag. & 25 & $4(16,00 \%)$ \\
\hline Lucan. & 22 & $1(4,54 \%)$ \\
\hline Stat., Theb ${ }^{49}$. & 23 & $5(21,73 \%)$ \\
\hline TOTAL poesía & 277 & $33(11,91 \%)$ \\
\hline TOTAL GLOBAL & 481 & $54(11,22 \%)$ \\
\hline
\end{tabular}

La ampliación del corpus de análisis lleva a que las cantidades porcentuales de utilización de la estructura de maneo con dativo prácticamente se equilibren. Aunque, en efecto, en construcciones tan escasas las bajas cifras que se obtienen en los sucesivos análisis recomienden mantener la prudencia, lo cierto es que con lo que muestran los datos con los que se cuenta, no es

${ }^{46}$ Cf. nota 5.

${ }^{47}$ Recuérdese que las cantidades aparecen incrementadas con los ejemplos correspondientes a la totalidad de la obra tacitea: 51 casos más, de los que $6(=11,76 \%)$ corresponden a construcciones en dativo. Estos datos confirman que el autor es constante en su utilización de la construcción en toda su obra de manera general.

${ }^{48}$ A los datos del corpus ampliado se suman los de Ou., Met.

49 Los casos de maneo en Achil. son muy escasos (5x) y ninguno de ellos se encuentra construido con dativo. 
posible confirmar de una forma clara la aludida ${ }^{50}$ preferencia de los poetas por la construcción con dativo. Si bien una investigación pormenorizada y aún más amplia ${ }^{51}$ podría desvelar tendencias más nítidas, es posible que la utilización de este tipo de construcción responda más bien a las preferencias y el estilo de los distintos autores y no se encuentre obligatoriamente vinculada a la forma poética de sus escritos.

\section{Test DEL VALOR NOCIONAL Y DE LAS CARACTERÍSTICAS DE LA CONSTRUCCIÓN}

Una vez aquilatada la cuestión del empleo del dativo en relación con el tipo específico de corpus, cabe preguntarse si es posible proponer un test que trate de confirmar el valor existencial-posesivo más y menos prototípico propuesto para estas estructuras. Pues bien, si, como se ha sugerido, se trata de construcciones similares a las que aparecen con el verbo copulativo por excelencia - y que de hecho se manifiestan en ejemplos como (11) y (14b) - , tendrían que compartir, en mayor o menor medida, los rasgos que exhiben esas construcciones ${ }^{52}$. Así, si se seleccionan algunas de esas características $^{53}$, obtenemos los resultados que recoge la Tabla 5, en los que sólo se ofrecen cantidades porcentuales en los totales, ya que el número de apariciones es, como se ha visto, escaso ${ }^{54}$ :

${ }^{50}$ García-Hernández 1996, p. 36. Recuérdese que, en el corpus de REGLA, los casos comentados con preverbados aparecen todos en prosa; en la ampliación del corpus, existen 22 casos de preverbados en poesía, ninguno de ellos con dativo.

51 Trabajo que se realizará en otro lugar.

${ }^{52} \mathrm{La}$ comparación es pertinente en cuanto que se encuentran documentados ejemplos paralelos como los siguientes: relictum a consule exercitum Q. Fuluius proconsul acciperet eique in annum imperium esset («se le asignó una legión que el año anterior había estado bajo el mando de Fulvio», Liu. XXVII 35.14) - neque mansurum Tiberio imperium si iis quoque legionibus cupido nouandi fuisset («y de que Tiberio no habría conservado el poder si también estas legiones hubieran decidido amotinarse», Tac., Ann. IV 18.2); nulli sua forma manebat («para nadie permanecía su propia figura», Ou., Met. I 5) - forma uiro, quam cernis, erat («el hombre tenía la figura que ves», Ou., Met. XIV 322).

${ }^{53} \mathrm{La}$ aplicación de todas ellas no resulta posible no sólo por cuestiones de espacio, sino porque el corpus incluye textos en prosa y en poesía, en los que aspectos como el orden de constituyentes o las secuencias pragmáticas pueden verse condicionados.

${ }^{54}$ En todo caso, la totalidad de casos analizados de maneo, permaneo, y remaneo asciende a 703 ejemplos. 
Tabla 5. Rasgos de las estructuras existencial-posesivas ${ }^{55}$ (maneo, permaneo y remaneo).

\begin{tabular}{|l|c|c|c|c|c|}
\hline \multicolumn{1}{|c|}{ Corpus } & $\begin{array}{c}\text { Dativo } \\
\text { [+humano] }\end{array}$ & $\begin{array}{c}\text { Dat. pronominal } \\
\text { (conocido) }^{56}\end{array}$ & $\begin{array}{c}\text { A1 } \\
\text { [+abstracto] }\end{array}$ & $\begin{array}{c}\text { 'Posesión' } \\
\text { ocasional }\end{array}$ & $\begin{array}{c}\text { 'Posesión' } \\
\text { con [-control] }\end{array}$ \\
\hline $\begin{array}{l}\text { Corpus prosa- } \\
\text { maneo (21x) }\end{array}$ & $21 \mathrm{x}$ & $4 \mathrm{x}$ & $21 \mathrm{x}$ & $18 \mathrm{x}$ & $19 \mathrm{x}$ \\
\hline $\begin{array}{l}\text { Corpus prosa- } \\
\text { preverbados (5x) }\end{array}$ & $3 \mathrm{x}$ & $5 \mathrm{x}$ & $5 \mathrm{x}$ & $1 \mathrm{x}$ & $4 \mathrm{x}$ \\
\hline $\begin{array}{l}\text { Corpus poético } \\
\text { ampliado (33x) }\end{array}$ & $32 \mathrm{x}$ & $23 \mathrm{x}$ & $27 \mathrm{x}$ & $19 \mathrm{x}$ & $33 \mathrm{x}$ \\
\hline TOTAL (59x) & $56(94,91 \%)$ & $32(54,23 \%)$ & $53(89,83 \%)$ & $38(64,49 \%)$ & $56(94,91 \%)$ \\
\hline
\end{tabular}

La tabla 5 informa de que las características analizadas en el verbo sum son compartidas por los tres verbos bajo estudio en más de la mitad de las ocasiones o en su práctica totalidad. Más concretamente, y si se comparan los resultados obtenidos con los que se observan en el análisis de un corpus de prosa - la totalidad de la obra de Livio - con el verbo sum, se comprueba que las proporciones son, en general, muy similares; las diferencias más significativas podrían deberse al diferente tipo de texto analizado:

Tabla 6. Rasgos de las estructuras existencial-posesivas (sum y verbos de 'permanencia').

\begin{tabular}{|c|c|c|c|c|c|}
\hline Corpus & $\begin{array}{c}\text { Dativo } \\
\text { [+humano] }\end{array}$ & $\begin{array}{c}\text { Dat. pronominal } \\
\text { (conocido) }^{59}\end{array}$ & $\begin{array}{c}\text { A1 } \\
\text { [+abstracto] }\end{array}$ & $\begin{array}{c}\text { 'Posesión' } \\
\text { ocasional }\end{array}$ & $\begin{array}{c}\text { 'Posesión' } \\
\text { con [-control] }\end{array}$ \\
\hline Liu. (185x) & $174(94,05 \%)$ & $77(41,62 \%)$ & $149(80,54 \%)$ & $138(74,59 \%)$ & $125(67,56 \%)$ \\
\hline Tabla 5 (59x) & $56(94,91 \%)$ & $32(54,23 \%)$ & $53(89,83 \%)$ & $38(64,49 \%)$ & $56(94,91 \%)$ \\
\hline
\end{tabular}

${ }^{55}$ Recopiladas y aplicadas en Cabrillana 2003.

${ }^{56}$ La proporción de dativos con naturaleza pragmática de información conocida subiría significativamente si no se hubieran computado sólo las formas pronominales.

${ }^{57} \mathrm{Al}$ corpus de REGLA se añaden, de nuevo, las cantidades correspondientes a la obra tacitea completa y se sustraen las cantidades de Ou., Met. que pasan a consignarse en el total del corpus poético.

${ }^{58}$ Corresponden exclusivamente a maneo; sólo existe un ejemplo de preverbado con dativo en corpus poético: Sen., Med. 42: per uiscera ipsa quaere supplicio uiam, / si uiuis, anime, si quid antiqui tibi / remanet uigoris («en las mismas entrañas busca el camino para la venganza, si estás viva, alma mía, si algo te queda de tu antiguo valor»).

${ }^{59} \mathrm{Cf}$. lo anotado al respecto en la Tabla 5. 
Un apoyo más de la posible naturaleza existencial-posesiva de las construcciones bajo estudio, es la existencia de casos en los que aparece la estructura en cuestión coordinada con una oración en la que se emplea uno de los verbos que expresan la posesión por excelencia, y ello en la descripción de unas realidades paralelas:

(15) obscuraque fronti / uitta manet, ramumque tenet morientis oliuae (Stat., Theb. VIII 89)

the fillet remains on his brow, albeit dim, and he holds the branch of dying olive

Podría aducirse, sin embargo, que se trata de un ejemplo «atípico» ya que el A1 de la construcción con dativo no es [+abstracto] sino [+concreto], lo cual rompe un tanto la tendencia; ahora bien, este mismo hecho podría ser síntoma de que la construcción va 'cristalizando' hacia la expresión de una 'posesión’ cada vez más concreta.

\section{Conclusiones. Características léXicas, ESQUEMA DE COMPLEMENTACIÓN Y CONSTITUCIÓN DE LA ESTRUCTURA}

Vistas estas características y lo anteriormente mostrado, sería posible ya proponer un esquema de complementación, en términos prototípicos, para las construcciones de los verbos analizados en este estudio. Se trataría de una estructura biargumental cuya segunda casilla se actualiza sólo eventualmente ${ }^{60}$ :

(16) A1 [-concreto $]_{\text {Estativo }}{ }^{61}\left[\mathrm{~A} 2[\text { +animado }]_{\text {Receptor }}^{62}\right]$

Definición del contenido: "continuar existiendo [para alguien]"

No hay que perder de vista que Pinkster 1988 había ya tratado casos en los que el verbo tiene un A2 que no es acusativo, partiendo del principio de que los argumentos que no van en acusativo son propios de estados de cosas

${ }^{60}$ Es lo que señala el «[]» dentro del que se sitúa el A2.

${ }^{61}$ Se trata de la Función que Van Valin y LaPolla 1997, pp. 141-146 aplican a los Sujetos de Estados y Procesos. No obstante, en otras perspectivas se optaría por adjudicar la Función Semántica de Posesor.

${ }^{62}$ Con respecto a la dificultad de identificar la Función Semántica de estos dativos, cf. Baños 2009, pp. 187-188. 
no controlados y viceversa (Pinkster 1988, pp. 236-237). Este lingüista 1988, p. 240 ofrece además unas tendencias para la lengua latina: con verbos de tres posiciones, el dativo es preponderantemente humano, pero con verbos de dos posiciones, eso es sólo una ligera preferencia; pues bien, en los verbos estudiados aquí - y ello es coherente con las características de la construcción existencial-posesiva-, esta preferencia se convierte prácticamente en regla: sólo existen dos excepciones, en que el dativo es [+animado] y [+concreto] respectivamente ${ }^{63}$.

Creo que se puede asumir y aplicar aquí una de las conclusiones de este trabajo de Pinkster 1988, pp. 243-244: que la presencia del dativo como A2 (sobre todo en verbos compuestos) pueda ser explicada diacrónicamente, de modo que verbos que podían ser monovalentes o bivalentes en los que la segunda casilla frecuentemente no se ocupaba, se asociaban habitualmente a un dativo que expresaba 'interés', el cual dativo fue uniéndose progresivamente al verbo hasta convertirse en un A2: es el proceso que han podido seguir los verbos con valor existencial aquí analizados.

Con todo, no es desdeñable ni excluyente una explicación de carácter analógico-tipológico: la construcción de maneo con dativo sería analógica con la del dativo que se construye con sum, un predicado que es fundamental y prácticamente universal.

\section{BibLIOGRAFÍA}

Álvarez Huerta, O. 2009: «Acusativo», en Baños, J. M. (coord.), Sintaxis del latín clásico, Madrid, Liceus, pp. 131-154.

Baldi, Ph. y Nuti, A. 2010: «Possession», en Baldi, Ph. y Cuzzolin, P. (eds.), New perspectives on historical Latin syntax. Volume 3: Constituent Syntax: Quantification, Numerals, Possession, Anaphora, Berlín y Nueva York, pp. 239-387.

Baños, J. M. 2009: «Dativo», en Baños, J. M. (coord.), Sintaxis del latín clásico, Madrid, Liceus, pp. 185-209.

Baños, J. M., Cabrillana, C., Torrego, M. E. y de la Villa, J. (eds.) 2003: Praedicatiua. Complementación en griego y en latín, Anejo 53 de Verba, Santiago de Compostela.

${ }^{63}$ Cf. ej. (8) y la nota realizada al respecto, y Stat., Theb. VI 530: hinc uice iusta / gloria mansit equo («so in fair division the horse kept his glory»); en este último caso, al referente del dativo se le están atribuyendo de alguna manera características propias de una entidad [+humana]. 
Blake, B. J. 2001: Case, Cambridge.

Cabrillana, C. 2003: «Estudio de rasgos diferenciales en las estructuras de Genitivo y Dativo 'posesivos' en latín», en Baños, J. M., Cabrillana, C. M., Torrego, E. y de la Villa, J. (eds.), Praedicatiua. Complementación en griego y en latín, Anejo 53 de Verba, Santiago de Compostela, pp. 79-109.

Cabrillana, C. 2010: Consideración sintáctico-semántica de esse. Un estudio a través de la prosa de Livio, Santiago de Compostela.

Cabrillana, C. 2011: «Gradualidad en los procesos de transitivación de compuestos preverbados latinos: ilustración sobre verbos en $d \bar{e}-\mathrm{y}$ ex-», en García Blanco,

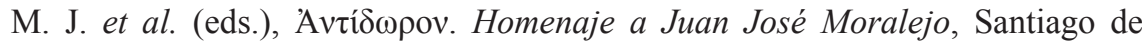
Compostela, pp. 95-110.

Cabrillana, C. 2015: «The argument/satellite distinction and absolute verbal use in Latin stative verbs», Journal of Latin Linguistics, 14(2), pp. 197-239.

Dik, S. C. 1989: The Theory of Functional Grammar. Part I: The structure of the clause, Dordrecht.

Ernout, A. y Meillet, A. 1932 (2001): Dictionnaire étymologique de la langue latine, París.

Ernout, A. y Thomas, F. 1953 (1989): Syntaxe Latine, París.

Forcellini, E. 1940: Totius Latinitatis Lexicon, Padua.

Gaffiot, F. 1991: Dictionnaire latin-français, París.

García-Hernández, B. 1996: «Modificación prefijal y régimen sintáctico. El testimonio de Arusiano Mesio», en Risselada, R., de Jong, J. R. y Bolkestein, A. M. (eds.), On Latin. Linguistic and Literary Studies in Honour of Harm Pinkster, Ámsterdam, pp. 25-43.

Glare, P. G. W. (ed.) 1982: Oxford Latin Dictionary, Oxford.

LASLA: <http://www.cipl.ulg.ac.be/Lasla/> (02/2015).

Lehmann, Ch. 1983: «Latin preverbs and cases», en Pinkster, H. (ed.), Latin linguistics and linguistic theory, Ámsterdam, pp. 145-161.

Lehmann, Ch. 2002: «Latin valency in typological perspective», en Bolkestein, A. M. et al. (eds.), Theory and description in Latin linguistics, Ámsterdam, pp. 183-203.

Longrée, D. 2005: «Verbes préfixés et concurrences syntaxiques chez Tacite», en Moussy, Cl. (ed.), La composition et la préverbation en latin, París, pp. 293-307.

Næss, A. 2009: «Varieties of dative», en Malchukov, A. y Spencer, A. (eds.), The Oxford Handbook of Case, Oxford, pp. 572-580.

Pinkster, H. 1988: «Non-accusative second arguments of two-place verbs in Latin», CFC-Elat 21, pp. 235-245.

Pinkster, H. 1995: Sintaxis y Semántica del latín, Madrid.

Pinkster, H. 2005: «The Language of Pliny the Elder», en Reinhardt, T., Lapidgey, M. y Adams, J. N. (eds.), The Language of Latin Prose, Oxford, pp. 239-256. 
Pinkster, H. 2010: «The use of the dative with Latin compounds», STUF - Language Typology and Universals / Sprachtypologie und Universalienforschung, 63, pp. 32-43.

Pinkster, H. 2015: Oxford Latin Syntax. I: The Simple Clause, Oxford.

Rosén, H. 1998: «Latin presentational sentences», en García-Hernández, B. (ed.), Estudios de lingüística latina, II, Madrid, pp. 723-742.

Seiler, H. 1983: Possession as an operational dimension of language, Tubinga.

Thesaurus Linguae Latinae, 1904-: Lipsia.

Torrego, M. E, Baños, J. M., Cabrillana, C. y Méndez Dosuna, J. (eds.) 2007:

Praedicatiua II: Esquemas de complementación verbal en griego antiguo y latín, Zaragoza.

Van Valin, R. D. y LaPolla, R. J. 1997: Syntax: structure, meaning and function, Cambridge. 